\title{
Phosphorylation of the ASF/SF2 RS domain affects both protein-protein and protein-RNA interactions and is necessary for splicing
}

\author{
Shou-Hua Xiao and James L. Manley ${ }^{1}$ \\ Columbia University, Department of Biological Sciences, New York, New York 10027 USA
}

\begin{abstract}
ASF/SF2 is a member of a conserved family of splicing factors known as SR proteins. These proteins, which are necessary for splicing in vitro, contain one or two amino-terminal RNP-type RNA-binding domains and an extensively phosphorylated carboxy-terminal region enriched in repeating Arg-Ser dipeptides (RS domains). Previous studies have suggested that RS domains participate in protein-protein interactions with other RS domain-containing proteins. Here we provide evidence that the RS domain of unphosphorylated recombinant ASF/SF2 is necessary, but not sufficient, for binding to the U1 snRNP-specific $70-\mathrm{kD}$ protein $(70 \mathrm{~K})$ in vitro. An apparent interaction of the isolated RS domain with $70 \mathrm{~K}$ was observed if contaminating RNA was not removed, suggesting a nonspecific bridging between the basic RS domain, RNA, and 70K. In vitro phosphorylation of recombinant ASF/SF2 both significantly enhanced binding to $70 \mathrm{~K}$ and also eliminated the RS domain-RNA interaction. Providing evidence that these interactions are relevant to splicing, ASF/SF2 can bind selectively to U1 snRNP in an RS domain-dependent, phosphorylation-enhanced manner. We also describe conditions that reveal for the first time a phosphorylation requirement for ASF/SF2 splicing activity in vitro.
\end{abstract}

[Key Words: ASF/SF2; SR proteins; RS domain; phosphorylation; pre-mRNA splicing]

Received November 14, 1996; revised version accepted December 18, 1996.

Pre-mRNA splicing is catalyzed in the spliceosome, which is formed by the assembly onto the pre-mRNA of five small ribonucleoprotein particles [U1, U2, U4/U6, and U5 (snRNPs)] and many non-snRNP proteins (for review, see Green 1991; Moore et al. 1993; Krämer 1996). Recognition of splice sites first occurs during formation of the commitment complex, the earliest event in spliceosome assembly. The commitment complex was originally defined in yeast pre-mRNA splicing, where in vitro processing of pre-mRNA assembled in a pre-spliceosomal complex was found to be resistant to an excess of competitor RNA added subsequently to the reaction (Ruby and Abelson 1988; Seraphin and Rosbash 1989). U1 snRNP, but no other snRNP, is present in the complex. In mammalian splicing extracts, commitment activity was shown in the pre-spliceosomal E complex (Michaud and Reed 1991; Jamison et al. 1992). U1 snRNP is again the only snRNP present in this complex, under-

${ }^{1}$ Corresponding author. FAX (212) 865-8246. scoring the importance of the U1 snRNP-pre-mRNA interaction.

Recognition of the $5^{\prime}$ splice site has long been known to involve base-pairing between the $5^{\prime}$-end of U1 snRNA and the $5^{\prime}$ splice site (Zhuang and Weiner 1986; Seraphin et al. 1988; Siliciano and Guthrie 1988). Although yeast $5^{\prime}$ splice sites are nearly invariant, their counterparts in higher eukaryotes show considerable variation (e.g., Mount 1982), suggesting that the metazoan U1 snRNP$5^{\prime}$ splice site interaction requires factor(s) in addition to base-pairing. Recent studies have suggested that members of the SR protein family can perform this function. Individual SR proteins were shown to be sufficient to commit pre-mRNAs to splicing, suggesting that these proteins play an important role in the formation of commitment complexes (Fu 1993). In addition, the SR protein ASF/SF2 interacts cooperatively with purified U1 snRNP to form stable complexes on 5' splice site-containing RNAs (Kohtz et al. 1994; Jamison et al. 1995). Consistent with this, addition of SR proteins to splicingcompetent nuclear extracts can enhance $5^{\prime}$ splice site 
occupation by U1 snRNP (Eperon et al. 1993; Zahler and Roth 1995).

The SR proteins constitute a family of splicing factors that are highly conserved in metazoa, and have been studied extensively in vitro (for review, see Fu 1995; Manley and Tacke 1996; Valcárcel and Green 1996). Individual SR proteins contain one or two amino-terminal RNP-type RNA-binding domains (RBD) and a carboxyterminal region of variable length that is enriched in repeating Arg-Ser dipeptides (RS domain). SR proteins have been shown to influence splicing in vitro in multiple ways: They are essential for splicing of all introns tested (e.g., Krainer et al. 1990a 1991; Ge et al. 1991; Fu and Maniatis 1992; Zahler et al. 1992); their concentration can influence the selection of competing splice sites in alternatively spliced pre-mRNAs (e.g., Ge and Manley 1990; Krainer et al. 1990b; Zahler et al. 1993); and they are required for the activity of splicing enhancer elements, which are typically exonic sequences capable of activating the splicing of weak upstream introns (e.g., Sun et al. 1993; Tian and Maniatis 1993; Staknis and Reed 1994).

Recent studies have provided insights into the molecular interactions involved in SR protein function. In the case of ASF/SF2, the two RBDs appear necessary and sufficient for binding pre-mRNA, and this region of the protein is also sufficient to induce concentration-dependent switching of alternative splice sites when added to extracts containing endogenous SR proteins (Cáceres and Krainer 1993; Zuo and Manley 1993). ASF/SF2 can recognize certain 5' splice sites (Zuo and Manley 1994) and a purine-rich sequence that matches a consensus found in a number of splicing enhancer elements (Sun et al. 1993; Tacke and Manley 1995). The RS domain of ASF/ SF2 is necessary for the protein to function as an essential factor in vitro (Cáceres and Krainer 1993; Zuo and Manley 1993) and for cell viability in vivo (Wang et al. 1996). Several experiments suggest that this region acts as a protein interaction domain. Wu and Maniatis (1993) provided evidence that ASF/SF2 and the SR protein SC35 interact both with each other as well as with the Ul snRNP-specific 70K protein (70K), and data that the RS domain of SC35 is necessary for interaction was described. Kohtz et al. (1994) also presented data supporting the interaction between ASF/SF2 and $70 \mathrm{~K}$, and showed that the RS domain of ASF/SF2 and a related region in $70 \mathrm{~K}$ are necessary for interaction. Given that the ASF/SF2 RS domain is also required for cooperation with U1 snRNP in $5^{\prime}$ splice site binding (Kohtz et al. 1994; Jamison et al. 1995), these findings support a model in which RS domain-mediated protein-protein interactions function in $5^{\prime}$ splice site recognition by $\mathrm{U} 1$ snRNP and SR proteins.

SR proteins are known to be phosphorylated, predominantly on serines in the RS domain (e.g., Roth et al. 1991; Colwill et al. 1996). Evidence has been presented that both phosphorylation and dephosphorylation are necessary for splicing. Addition of the phosphatase PP1 to nuclear extracts inhibits spliceosome assembly, perhaps by dephosphorylating endogenous SR proteins (Mer- moud et al. 1994). In contrast, addition of phosphatase inhibitors also blocks splicing but at a step subsequent to spliceosome assembly (Mermoud et al. 1992; Tazi et al. 1992). This could perhaps reflect a requirement for dephosphorylation of 70K (Tazi et al. 1993) and/or SR proteins. Finally, Cardinali et al. (1994) showed that phosphatase treatment of nuclear extracts could influence alternative splice site selection, reminiscent of effects of SR proteins. Thus, there is considerable evidence that SR proteins are phosphorylated in their RS domain, and it is likely that both phosphorylation and dephosphorylation play important roles in splicing and/or its regulation. However, nothing is known about the molecular interactions influenced by RS domain phosphorylation.

Here we provide new insights into the function of the ASF/SF2 RS domain and the effects of phosphorylation on its activity. We show that the ASF/SF2 RS domain is necessary but not sufficient for binding to $70 \mathrm{~K}$. Phosphorylation of the RS domain not only prevents nonspecific interactions with RNA, which otherwise can "bridge" the two proteins in a perhaps nonphysiological manner but also significantly enhances RNA-independent protein-protein interaction. Supporting the relevance of these interactions to splicing, we show that ASF/SF2 can interact selectively with U1 snRNP and that this interaction is also RS domain-dependent and enhanced by phosphorylation. We also provide evidence that phosphorylation can be essential for ASF/SF2 to function in splicing.

\section{Results}

The RS domain of ASF/SF2 is necessary but not sufficient for binding U1 $70 \mathrm{~K}$

ASF/SF2 was shown previously to interact in vitro with 70K by Far Western blotting and coimmunoprecipitation of the in vitro-translated proteins, as well as by yeast two-hybrid assays (Wu and Maniatis 1993; Kohtz et al. 1994). The RS domain of ASF/SF2 and a related region in $70 \mathrm{~K}$ were found to be necessary for the interaction in Far Western assays, whereas small deletions elsewhere in ASF/SF2 were without effect (Kohtz et al. 1994), raising the possibility that the RS domain might be sufficient for association. To gain more insights into this interaction, we adapted the glutathione $S$-transferase (GST) fusion protein interaction assay. Recombinant plasmids encoding GST-ASF/SF2 (GST-ASF) and derivatives containing the RS domain (GST-RS) or the remainder of the protein (GST- $\Delta$ RS) were constructed (Fig. 1B) and the recombinant proteins purified from Escherichia coli (see Fig. 2A). The fusion proteins, as well as purified GST itself, were used in binding assays with $\left[{ }^{35} \mathrm{~S}\right]$-methionine-labeled $70 \mathrm{~K}$ produced by in vitro transcription-translation, and bound proteins were analyzed by SDS-gel electrophoresis (see Materials and Methods). Preliminary experiments failed to reveal significant differences whether bound proteins were eluted with glutathione or directly with SDS gel buffer (results not shown), and the latter method was therefore used in all experiments described 
A

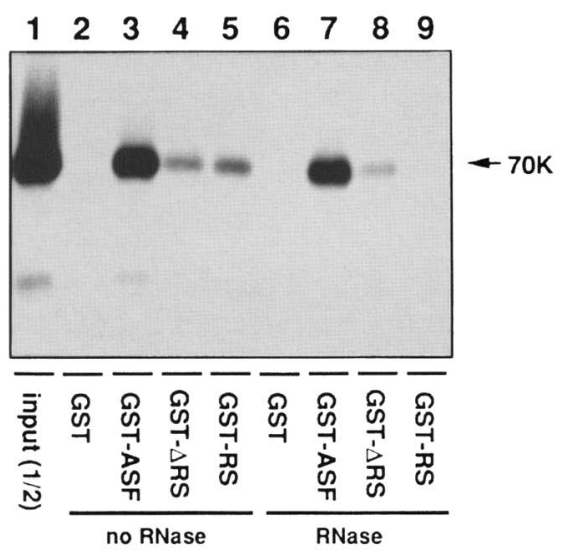

B

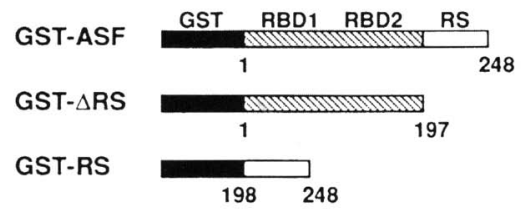

Figure 1. The RS domain of ASF/SF2 is necessary but not sufficient for interaction with U1 70K protein. (A) GST binding assay. GST, GST-ASF, GST- $\Delta$ RS, and GST-RS (diagramed in $B$ ) were purified from $E$. coli. Three micrograms of each fusion protein was bound to glutathione-agarose beads and used in binding assays with $\left[{ }^{35} \mathrm{~S}\right]$ methionine-labeled U1 $70 \mathrm{~K}$. The samples indicated were treated with RNase prior to elution. Bound proteins were eluted and analyzed by $7 \%$ SDS-PAGE followed by fluorography. The position of $70 \mathrm{~K}$ is indicated. $(B)$ Structures of GST fusion proteins. The GST moiety, RBDs, and RS domains are indicated. Numbers indicate ASF/SF2 amino acid residues.

here. Figure 1A shows the results of a typical binding experiment. Most notably, very efficient binding of $70 \mathrm{~K}$ to GST-ASF was detected (lane 3). 70K also bound the two mutant derivatives, but in each case the interaction was much weaker than observed with GST-ASF (lanes 4,5 ). $70 \mathrm{~K}$ did not bind detectably either to GST (lane 2) or to a heterologous GST-TFIIB fusion protein (results not shown). Together, these results provide further evidence that ASF/SF2 and 70K interact specifically, and that the RS domain of ASF/SF2 is necessary for efficient binding.

Both ASF/SF2 and 70K are RNA-binding proteins, and it was thus conceivable that the interactions just described were mediated or enhanced by RNA in the reaction mixtures (e.g., see Lutz et al. 1996). Such RNA could have come from the reticulocyte lysate and/or as a contaminant bound to the proteins isolated from E. coli. This hypothetical bridging RNA could associate with the proteins' RBDs via weak, nonspecific interactions or, in the case of GST-RS, with the very basic RS domain (which would be unphosphorylated in E. coli) via ionic interactions. To test this possibility, binding reactions identical to those described above were performed, ex- cept that RNase A was added to reaction mixtures prior to washing and elution. The results (Fig. 1A, lanes 6-9) were striking: Although neither the efficient binding of $70 \mathrm{~K}$ to GST-ASF nor the weak binding to GST- $\Delta \mathrm{RS}$ was significantly affected by RNase treatment, binding to GST-RS was essentially abolished. These results provide strong support for the existence of a direct interaction between ASF/SF2 and 70K. In addition, although the RS domain of ASF/SF2 is necessary for binding, it is not sufficient and, by itself, is completely unable to interact with $70 \mathrm{~K}$ (see also below).

\section{Phosphorylation of the ASF/SF2 RS domain enhances binding to $U 170 \mathrm{~K}$}

We then wanted to investigate whether RS domain phosphorylation might affect the interactions with $70 \mathrm{~K}$ described above. As a first experiment, the three purified GST fusion proteins were phosphorylated by incubation in HeLa S100 extract under splicing conditions and then repurified by binding to glutathione-agarose followed by extensive washing (see Materials and Methods). For controls, aliquots of the fusion proteins were treated identically except that ATP was omitted from the S100 incubations (mock phosphorylation). To verify the phos-

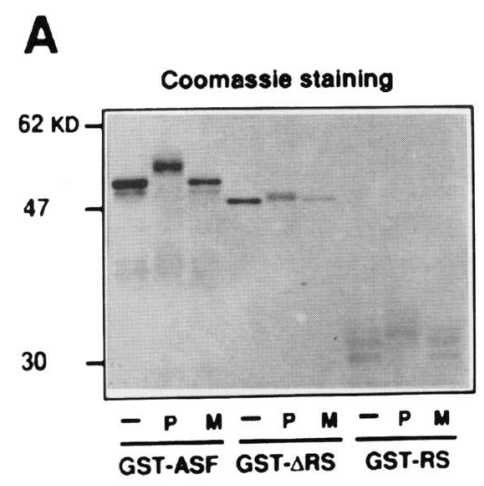

B

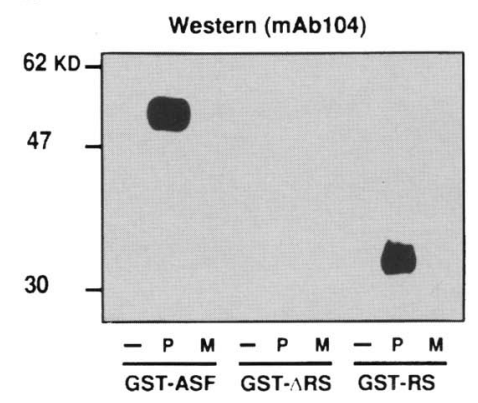

Figure 2. Phosphorylation of GST-ASF fusion proteins. GSTASF, GST- $\Delta$ RS, and GST-RS were untreated (-), phosphorylated (P), mock phosphorylated (M) in HeLa S100, repurified, and aliquots $(3 \mu \mathrm{g})$ were analyzed by SDS-PAGE. $(A)$ Coomassie staining; $(B)$ Western blot with mAb 104. The sizes of protein markers are indicated at left. The predicted molecular sizes of the GST fusions are GST-ASF (53 kD), GST- $\Delta$ RS (48 kD), and GST-RS (31 kD). 
phorylation status of the repurified proteins, aliquots were analyzed by SDS-gel electrophoresis and Western blotting. Coomassie staining (Fig. $2 \mathrm{~A}$ ) revealed that the mobilities of the GST-ASF and GST-RS proteins were significantly reduced following incubation in S100, and the mobility of GST- $\Delta$ RS was slightly reduced. In contrast, the mobilities of the mock-phosphorylated proteins were identical to the corresponding untreated proteins. In the case of GST-ASF, the mobility shift was shown to be the result of phosphorylation by the observation that it was eliminated by phosphatase treatment (results not shown). Evidence that the phosphorylation involved physiologically relevant sites in the RS domain was obtained by probing a Western blot with mAb 104, known to recognize a phosphoepitope in the RS domain (Fig. 2B; Roth et al. 1990). GST-ASF and GST-RS incubated in S100 with ATP, but none of the other samples, showed strong reactivity with mAb 104.

To determine the effects of the S100-induced phosphorylation on interactions with $70 \mathrm{~K}$, we performed binding reactions similar to those shown in Figure 1A, first in the absence of RNase treatment. The results (Fig. 3) allow several conclusions. First, phosphorylation enhanced the interaction between GST-ASF and $70 \mathrm{~K}$, as significantly more $70 \mathrm{~K}$ was bound to the phosphorylated fusion protein than to the mock-phosphorylated protein (cf. lanes 3 and 7). Second, mock-phosphorylated GSTRS bound $70 \mathrm{~K}$ as efficiently as mock-phosphorylated GST-ASF. This contrasts with the behavior of untreated GST-RS, which bound 70K significantly less efficiently than did GST-ASF (cf. Fig. 3, lanes 7 and 9, with Fig. 1A, lanes 3 and 5), and could reflect higher amounts of bridging RNA provided by the $\mathrm{S} 100$ extract. Third, phosphorylation of GST-RS abolished interaction with $70 \mathrm{~K}$ (cf. lanes 5 and 9). This likely reflects inhibition of the RSRNA interaction, with phosphorylation reducing the net positive charge of the RS domain and thereby interfering with the apparent ionic interaction between the RS domain and RNA. Finally, phosphorylation had no signifi-

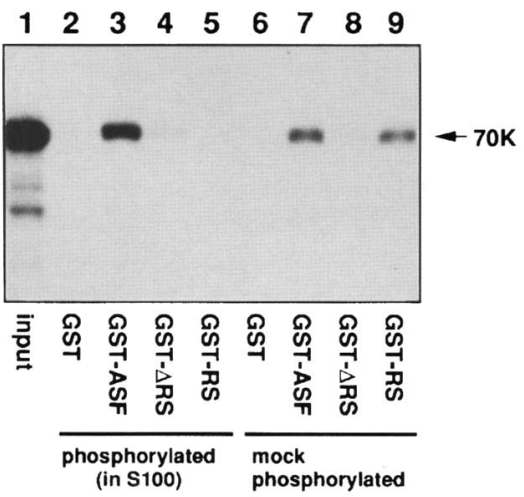

Figure 3. Phosphorylation of GST-ASF fusion proteins affects binding to $\mathrm{U} 170 \mathrm{~K}$. The indicated phosphorylated and mockphosphorylated fusion proteins (described in Fig. 2) were used in binding reactions with $70 \mathrm{~K}$ (without RNase) and analyzed exactly as in Fig. 1.
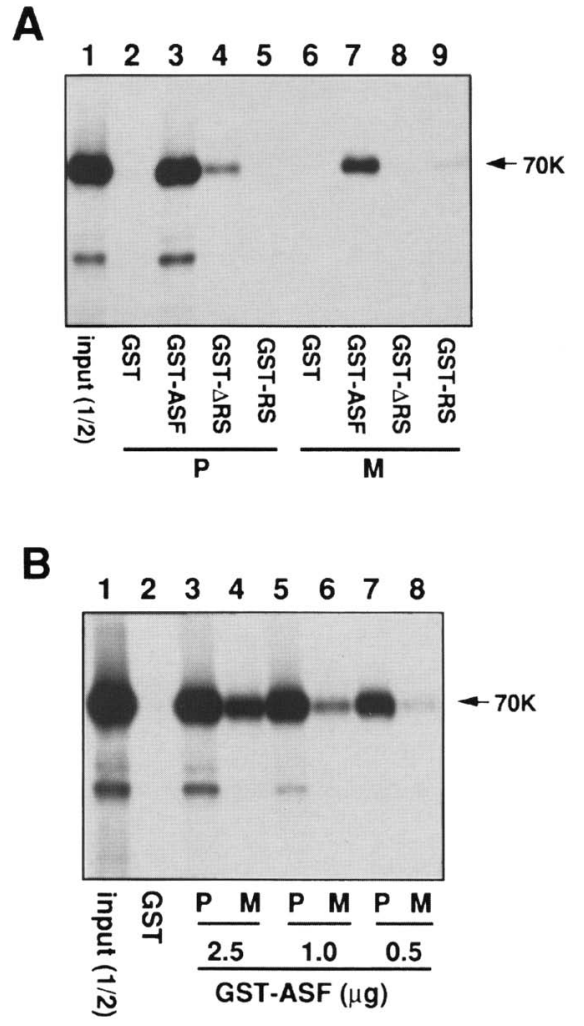

Figure 4. Phosphorylation enhances the ASF/SF2-70K interaction. $(A)$ Binding of phosphorylated $(\mathrm{P})$ and mock-phosphorylated (M) GST, GST-ASF, GST- - RS and GST-RS to U1 70K in the presence of RNase. Binding reactions and analysis were performed as in Fig. 1. (B) Phosphorylation enhancement of $70 \mathrm{~K}$ binding is increased at lower protein concentrations. The indicated amounts of phosphorylated $(\mathrm{P})$ and mock-phosphorylated (M) GST-ASF were used in binding reactions in the presence of RNase, as in Fig. 1, except that the amount of in vitro-translated U1 $70 \mathrm{~K}$ was doubled.

cant effect on the weak interaction between GST- $\Delta$ RS and $70 \mathrm{~K}$. We also determined the effect of RNase treatment on these interactions (Fig. 4A). The efficient binding of 70K to GST-ASF was essentially unaffected by RNase treatment, with phosphorylation again producing a significant increase in binding relative to that observed with the mock-phosphorylated protein (Fig. 4B, cf. lanes 5 and 9). The enhancement due to phosphorylation (about fivefold) was slightly higher than in the absence of RNase, reflecting a small decrease in the interaction of mock-phosphorylated GST-ASF with $70 \mathrm{~K}$ following RNase treatment. Not unexpectedly, the interaction between mock-phosphorylated GST-RS and 70K was essentially eliminated by RNase. Together, these results provide evidence that phosphorylation of the ASF/SF2 RS domain both enhances specific protein-protein interactions with $70 \mathrm{~K}$ and also prevents nonspecific binding to RNA.

Under the conditions employed in the above experiments, $50 \%$ or more of the input $70 \mathrm{~K}$ was bound by phosphorylated GST-ASF. This raised the possibility 
that the pool of free $70 \mathrm{~K}$ had become limiting during binding reactions, resulting in an underestimate of the effect of phosphorylation. To test this, binding reactions were performed as in Figure 4A, except with an increased concentration of $70 \mathrm{~K}$ and decreasing amounts of phosphorylated or mock-phosphorylated GST-ASF (Fig. 4B). Bound proteins were quantitated by PhosphorImager, which revealed that under these conditions phosphorylation enhanced binding up to 10 -fold (i.e., lanes 5-8).

\section{ASF/SF2-70K binding involves both ionic and nonionic interactions}

To gain insight into the nature of the interaction between GST-ASF and 70K, we measured the salt sensitivity of binding. Specifically, phosphorylated and mockphosphorylated fusion proteins were used in binding reactions identical to those shown in Figure 4A, except that the bound proteins were washed with buffer containing increasing concentrations of $\mathrm{NaCl}$ and eluted proteins were quantitated by PhosphorImager analysis of SDS gels. The results (Fig. 5) indicate that the enhancement in binding due to phosphorylation was sensitive to moderate salt concentrations. Washing with $0.5 \mathrm{M} \mathrm{NaCl}$ reduced binding fivefold, essentially eliminating the increased binding resulting from phosphorylation. However, the binding that remained was almost entirely salt resistant, with no significant decrease observed at $\mathrm{NaCl}$ concentrations as high as $1.5 \mathrm{M}$. Evidence that the saltsensitive binding involved phosphorylated residues was provided by the behavior of the mock-phosphorylated protein, which did not show a significant salt-sensitive

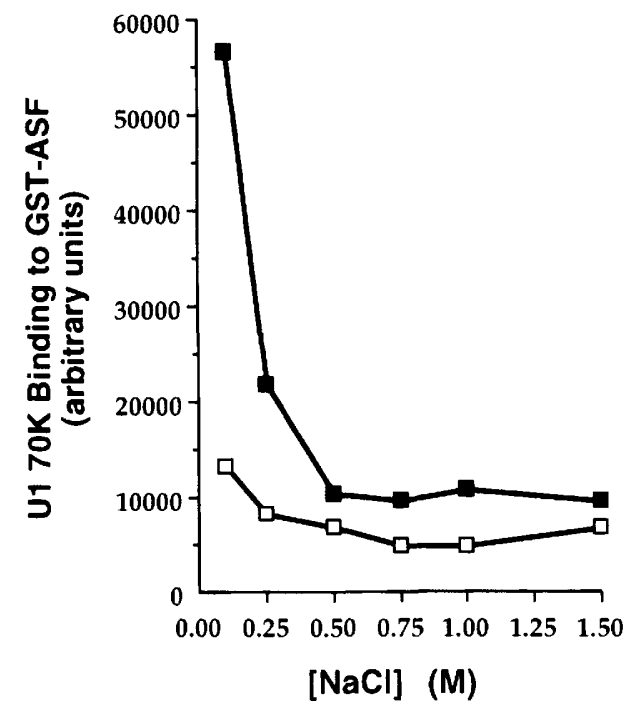

Figure 5. Salt sensitivity of the GST-ASF/Ul 70K interaction. After binding and RNase treatment as in Fig. 4A, bound proteins were washed three times with NETN containing the indicated $\mathrm{NaCl}$ concentration, followed by elution and analysis. The intensity of each band was quantitated by PhosphorImager analysis. (घ) Phosphorylated; ( $\square$ ) mock. The values shown are the means of three experiments. component. Only small reductions in binding were detected as the $\mathrm{NaCl}$ concentration was increased, with binding at $1.5 \mathrm{M} \mathrm{NaCl} \sim 60 \%$ the level observed at $0.1 \mathrm{M}$. These results indicate that the increased binding due to phosphorylation is largely ionic, likely involving the phosphoserine residues in the ASF/SF2 RS domain. However, a significant fraction of the phosphorylationindependent binding involves nonionic interactions.

Phosphorylation of GST-ASF by recombinant Clk/Sty also enhances $70 \mathrm{~K}$ binding

To confirm and extend our results indicating that GSTASF phosphorylated in S100 extract displays enhanced binding to $70 \mathrm{~K}$, we performed similar experiments except that a purified recombinant SR protein kinase was used instead of S100 to phosphorylate GST-ASF. Clk/ Sty kinase has been shown to interact with ASF/SF2 in a yeast two-hybrid assay, to phosphorylate ASF/SF2 extensively and almost exclusively in the RS domain, and to colocalize with splicing factors in vivo (Colwill et al. 1996). We utilized a GST-Clk/Sty fusion (ClkK) expressed in and purified from E. coli, and as a control an otherwise identical protein containing an inactivating mutation (K190R) in the kinase active site (ClkR). GSTASF was incubated with ClkK or ClkR and the proteins first analyzed by gel electrophoresis and Western blotting (Fig. 6A). ClkK induced a significant mobility shift in GST-ASF, perhaps more complete than observed with S100, whereas incubation with ClkR had no effect. Furthermore, the protein incubated with $\mathrm{ClkK}$, but not ClkR, displayed strong reactivity with $\mathrm{mAb} 104$, confirming RS domain phosphorylation.

To determine whether phosphorylation of GST-ASF by $\mathrm{ClkK}$ enhanced interaction with $70 \mathrm{~K}$, binding experiments similar to those described in Figure 4A /with RNase) were performed (Fig. 6B). Because ClkK and ClkR are also both GST fusion proteins and were thus not removed from GST-ASF following the phosphorylation reactions, controls in which GST-ASF was omitted from phosphorylation and binding reactions were also included. However, no binding of $70 \mathrm{~K}$ to either ClkK or ClkR was detected (lanes 2,3). More importantly, although binding of untreated and ClkR-treated GST-ASF to $70 \mathrm{~K}$ was similar (cf. lanes 5,7), ClkK-phosphorylated GST-ASF bound $70 \mathrm{~K}$ with significantly greater efficiency (lane 6). These results both provide additional evidence that RS domain phosphorylation enhances the ASF/SF2 interaction with $70 \mathrm{~K}$ and also indicate that phosphorylation by Clk/Sty is sufficient for this enhancement.

\section{Phosphorylation enhances a specific interaction between GST-ASF and U1 snRNP}

The experiments described above have provided evidence supporting the existence of an RS domain-dependent, phosphorylation-enhanced interaction between $70 \mathrm{~K}$ and ASF/SF2. However, 70K does not normally exist as a free protein but, rather, as a part of $\mathrm{U} 1 \mathrm{snRNP}$. If the 
A

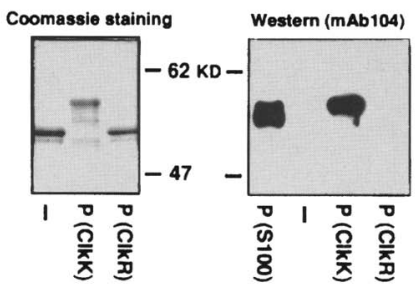

B

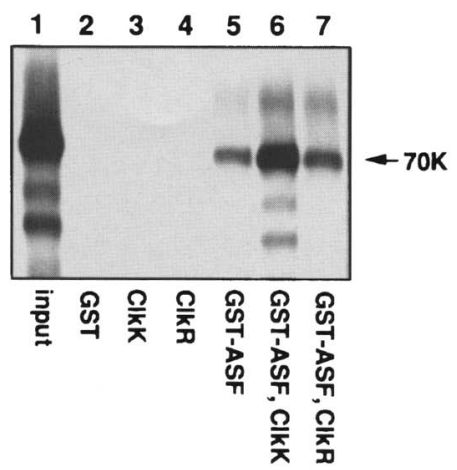

Figure 6. Phosphorylation of GST-ASF by Clk/Sty kinase enhances binding to U1 70K. (A) GST-ASF is phosphorylated by GST-ClkK, but not ClkR. Three micrograms aliquots of GSTASF incubated with the indicated kinase derivative were resolved by SDS-PAGE and analyzed by Coomassie staining (left) and Western blotting (right). GST-ASF phosphorylated by $\$ 100$ was included by the Western blot for comparison. $(B)$ Binding of Clk/Sty-phosphorylated GST-ASF to Ul 70K. GST-ASF was phosphorylated as in $A$ and then used in binding assays as in Fig. 4A. Assays with GST-ClkK or GST-ClkR contained $200 \mathrm{ng}$ of the indicated protein.

above interactions are physiologically relevant, then ASF/SF2 should be able to bind U1 snRNP and the interaction should be RS domain-dependent and enhanced by phosphorylation. To test this, purified GST, GSTASF, GS- $\Delta$ RS, and GST-RS were phosphorylated or mock-phosphorylated (using S100 extract) and repurified by binding glutathione-agarose and washing extensively. Binding reactions similar to those described above were then performed, except that HeLa S100 extract was used instead of in vitro-translated $70 \mathrm{~K}$, and binding was measured by Northern blotting of extracted RNAs, using U1, U2, and U4 snRNA probes (see Materials and Methods). The results, shown in Figure 7, allow several conclusions. First, GST-ASF efficiently bound Ul snRNP and, as with $70 \mathrm{~K}$, binding was significantly enhanced (about fourfold) by phosphorylation. Second, the interaction was highly selective: U2 snRNP binding was $<10 \%$ as efficient as Ul snRNP binding, and U4 snRNA was barely detectable (results with U4 not shown). Finally, GST- $\Delta$ RS and GST-RS bound U1 snRNP very weakly. Again analogous to the situation with $70 \mathrm{~K}$, the weak interaction between GST-RS and Ul snRNP was eliminated by phosphorylation. These results support the relevance of the ASF/SF2-70K interaction to a specific as- sociation between the SR protein and U1 snRNP, and also indicate that a function of RS domain phosphorylation is to enhance this interaction.

\section{Phosphorylation can be necessary for ASF/SF2 to function in splicing}

The above experiments, as well as previous work, suggest that the phosphorylation status of ASF/SF2 should influence its activity in splicing. To test this, His-tagged ASF/SF2 was purified from E. coli, phosphorylated or mock-phosphorylated with $S 100$, repurified under denaturing conditions, and renatured (Fig. 8A). Initial experiments failed to reveal significant differences between the two protein preparations in splicing assays, perhaps reflecting the fact that the mock-phosphorylated protein was rapidly phosphorylated following addition to nuclear or S100 extracts (results not shown; see below). In an effort to detect possible differences in the behavior of the two proteins at the earliest steps in the reaction, we modified the assay in two ways. First, an HIV-tat pre-mRNA substrate was used. This RNA is spliced only very poorly in nuclear extracts, except when supplemented with ASF/SF2 (Fig. 8B; Krainer et al. 1990a). Tat pre-mRNA can also be committed to splicing by ASF/ SF2 but not by other SR proteins (Fu 1993). Second, nuclear extracts were supplemented with random RNA. If nonphosphorylated ASF/SF2 binds nonspecifically to RNA under splicing conditions, it is possible that this would prevent its phosphorylation and/or interfere with its function in splicing.

The RNA products extracted from splicing reactions supplemented with mock or phosphorylated ASF/SF2 and increasing amounts of random RNA are shown in Figure 8C. [Note that the amounts of ASF/SF2 $(300 \mathrm{ng}$ ) and RNA (100-400ng) added were similar.] In the absence of random RNA, both forms of ASF/SF2 resulted in similar levels of splicing (lanes 1,2). However, in the presence of this RNA, the ability of mock-phosphory-

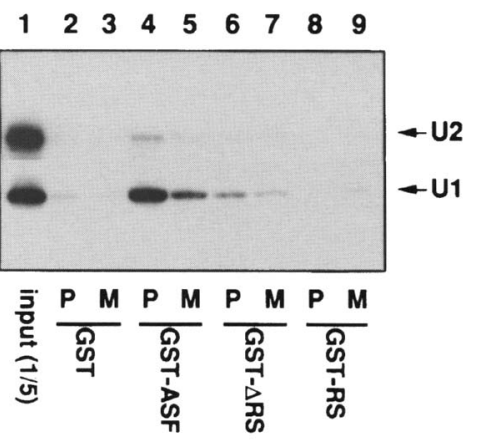

Figure 7. Phosphorylation of GST-ASF enhances its specific association with U1 snRNP. The indicated GST fusion proteins were phosphorylated or mock phosphorylated by incubation in HeLa S100, repurified, and used in binding reactions in which S100 extract was used in place of U1 70K. Eluted RNAs were purified and analyzed by Northern blotting with U1 and U2 snRNA probes. 


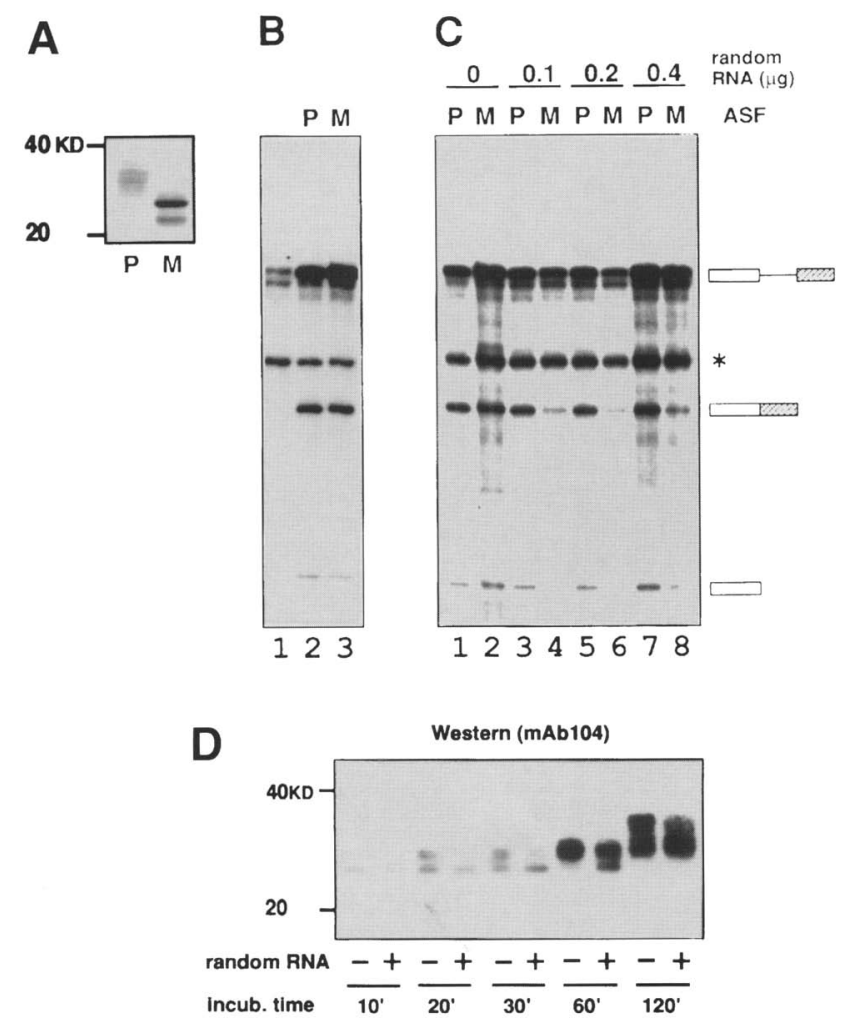

Figure 8. ASF/SF2 RS domain phosphorylation can be required for splicing in vitro. $\{A \mid$ Phosphorylation $(\mathrm{P})$ and mock phosphorylation $(M)$ of His-tagged ASF/SF2. His-ASF expressed in and purified from $E$. coli was phosphorylated or mock phosphorylated as described for GST-ASF in Fig. 2. Proteins were repurified by $\mathrm{Ni}^{++}$agarose chromatography, and aliquots $(3 \mu \mathrm{g})$ were analyzed by SDS-PAGE and Commassie staining. $(B)$ In vitro splicing of HIV tat pre-mRNA in nuclear extract supplemented with $300 \mathrm{ng}$ of phosphorylated $(\mathrm{P})$ or mock-phosphorylated (M) His-ASF. (Lane 1) Nuclear extract alone. RNAs were purified and analyzed by denaturing PAGE. $(C)$ Random RNA inhibits the splicing activity of mock- phosphorylated but not phosphorylated His-ASF. In vitro splicing of tat pre-mRNA was carried out as in $B$, except His-ASF (phosphorylated or mock phosphorylated) was preincubated with the indicated amounts of random RNA before addition to reaction mixtures. The positions of pre-mRNA, spliced mRNA, and $5^{\prime}$ exon are indicated schematically on the right. A species resulting from artifactual cleavage is indicated by an asterisk $\left(^{*}\right)$ (Krainer et al. 1990a; Fu 1993). (D) Random RNA inhibits phosphorylation of His-ASF. Mock-phosphorylated His-ASF was mixed with random RNA (400 ng) and then incubated in S100 under splicing conditions for the indicated time. Proteins were analyzed by Western blotting with $\mathrm{mAb} 104$.

lated, but not phosphorylated, ASF/SF2 to enhance splicing was reduced significantly (lanes 3-8). Evidence that inhibition by the mock-phosphorylated protein was related to its phosphorylation status was obtained by Western blotting with $\mathrm{mAb} 104$ of proteins extracted at different times from splicing reactions (employing S100) containing initially unphosphorylated ASF/SF2 plus or minus random RNA (Fig. 8D). With the exception of the first (10 min) time point, the extent of ASF/SF2 phosphorylation at all time points was significantly less in the presence of random RNA than in its absence. In contrast, the random RNA did not result in any decrease in the phosphorylation status of the phosphorylated ASF/ SF2 (data not shown). Together, our results establish that the phosphorylation status of ASF/SF2 influences the protein's ability to function in pre-mRNA splicing in vitro.

\section{Discussion}

The experiments described here have provided the first insights into the molecular mechanisms by which protein phosphorylation can affect SR protein activity and influence splicing. We have shown that RS domain phosphorylation has a dual function: It enhances protein-protein interactions while also preventing apparently nonspecific interactions with RNA. Below we discuss how these RS domain-mediated interactions, and their modulation by phosphorylation, can contribute to splicing and/or splicing control.

Our data have provided further support for the idea that an important function of the RS domain of ASF/ SF2-and by extension other SR proteins-is to participate in protein-protein interactions, specifically with the U1 $70-\mathrm{kD}$ protein. However, although the RS domain is necessary for this interaction, it is not sufficient, at least in the GST assay employed here. This was somewhat unexpected, given our previous inability to detect any regions outside the RS domain that were required for interaction in Far Western assays (Kohtz et al. 1994). However, this analysis was not exhaustive, so it is possible that the required region was not detected. Alternatively, there could be redundant non-RS regions, or the two assays might have different requirements. Whatever the case, the possibility of non-RS contacts raises an important question: Do RS domain-mediated interactions involve direct contact between RS domains, or does the RS domain of one protein (e.g., ASF/SF2) contact residues outside the RS region of its partner (e.g., 70K)? Although additional data are necessary to answer this question, we favor the view that the RS domains interact directly. All known SR-interacting proteins themselves contain an RS domain, and where studied this region has been shown to be essential for interaction (Wu and Maniatis 1993; Amrein et al. 1994; Kohtz et al. 1994; Colwill et al. 1996; Zhang and Wu 1996). The presence of an RS like domain is the only thing in common between these proteins, supporting the idea that binding involves direct contacts between RS domains. However, it is noteworthy that the interaction between ASF/SF2 and the Clk/Sty kinase, analyzed by two-hybrid assays, also requires $\mathrm{ASF} / \mathrm{SF} 2$ sequences in addition to the RS domain (Colwill et al. 1996).

How do highly charged RS domains interact, and how might phosphorylation enhance the interaction? Data presented here support the idea that RS domain-mediated interactions can occur in the absence of phosphorylation. We suggest that this involves ionic interactions 
between arginines in the ASF/SF2 RS domain and acidic residues in the 70K RS region (unlike RS domains in SR proteins, the $70 \mathrm{~K}$ RS region is enriched in RD and RE dipeptides). Following phosphorylation, such ionic interactions would be considerably strengthened by formation of phosphoserine-arginine pairs, which would be in keeping with the observed salt sensitivity of the phosphorylation-enhanced interaction. This structure could perhaps constitute a "polar zipper", as suggested recently by Perutz et al. (1993). These investigators proposed that chains of RD and RE repeats, as found in $70 \mathrm{~K}$, zip together to form an antiparallel $\beta$-sheet. Phosphorylation of serines in the ASF/SF2 RS domain could create a similar motif. However, whatever the precise nature of the ASF/SF2-70K structure, our data suggest that it is not mediated solely by RS domain interactions, as GST$\mathrm{RS}$ is unable to interact detectably with $70 \mathrm{~K}$. One possibility is that this reflects a strong interaction of ASF/ SF2 RS domains with each other, perhaps facilitated by dimerization of the GST moieties, which would leave the RS domains unavailable for interaction with $70 \mathrm{~K}$. By this view, the function of the non-RS region of ASF/SF2 would be to prevent this self-association. However, ASF/ SF2 has been shown to be capable of interacting with itself (Wu and Maniatis 1993), and it is therefore unlikely that the non-RS region would function to prevent such an interaction only in the context of the GST fusion protein. In addition, we detected a weak interaction between GST- $\Delta$ RS and 70K. This could function not only to help stabilize the association of the RS domains, perhaps providing much of the salt-resistant component of the interaction, but also to enhance its specificity.

Our data provide strong evidence that the unphosphorylated RS domain of ASF/SF2 can interact with RNA nonspecifically and that phosphorylation prevents this interaction. This finding is consistent with our recent results with another SR protein, SRp40, which showed that only the phosphorylated form could function in a SELEX experiment to identify a specific highaffinity binding site. The unphosphorylated protein bound the RNA avidly but could not select a specific sequence (Tacke et al. 1997). An important question is whether such RS domain-RNA interactions are physiologically relevant. A prerequisite for this is that unphosphorylated (or hypophosphorylated) SR proteins exist in vivo, an issue that remains to be investigated thoroughly. However, as mentioned in the introductory section, in vitro studies are consistent with the notion that serine/threonine dephosphorylation is necessary for splicing (but not spliceosome assembly). Dephosphorylation of SR proteins could allow conformational changes in the spliceosome by weakening protein-protein interactions while perhaps at the same time helping to "anchor" the pre-mRNA more stably in the spliceosome by allowing RS domain-RNA interactions. There is a precedent for RS domains participating in functionally important, nonsequence specific interactions with pre-mRNA. U2AF is a heterodimer that is necessary for recruitment for U2 snRNP to the pre-mRNA branch site. The large subunit $\mathrm{U}_{2} \mathrm{AF}^{65}$ contains carboxy-terminal
RBDs, which recognize the pyrimidine tract, usually located adjacent to the branchsite, and an essential aminoterminal RS region (Zamore et al. 1992). Recent studies have provided evidence that the RS region interacts with the pre-mRNA branch site in a way that facilitates basepairing with U2 snRNA (Valcárcel et al. 1996). Although there are significant differences between the $U 2 A F^{65}$ RS region and RS domains found in SR proteins [e.g., the former has not been implicated in protein-protein interactions; see Valcárcel et al. (1996) for further discussion], these results are consistent with the possibility that the ASF/SF2 RS domain-RNA interaction may have some functional significance. The possible effect of phosphorylation on the $\mathrm{U}_{2} \mathrm{AF}^{65}-\mathrm{RNA}$ interaction has not been investigated.

In addition to playing a functional role during the splicing cycle, RS domain phosphorylation could also regulate SR protein activity. For example, it is conceivable that unphosphorylated (or hypophosphorylated) SR proteins can accumulate under some situations and that these proteins are inactive in splicing. One possibility is that they reside in nuclear speckles, which are domains within the nucleus enriched in SR proteins and other splicing factors (for review, see Spector 1993). Current evidence suggests that speckles may be storage sites for splicing factors, and not the actual sites of splicing (e.g., Zhang et al. 1994). Thus, it is possible that underphosphorylated SR proteins accumulate in speckles and that nonspecific RS domain-RNA interactions help hold these structures together. Consistent with this, overexpression of either of two SR protein kinases, SRPK1 (Gui et al. 1994) or Clk/Sty (Colwill et al. 1996), is sufficient to cause release of SR proteins from the speckles, presumably because of RS domain phosphorylation.

Our data have provided the first demonstration that SR protein phosphorylation can be required for splicing. The design of our experiments allows two possible explanations, not mutually exclusive, for the reduced activity of unphosphorylated ASF/SF2. First, a phosphorylated RS domain is essential for one or more specific interactions that occur during splicing. Our data suggest that this includes the phosphorylation-enhanced interaction between ASF/SF2 and U1 snRNP, which is presumably mediated by the ASF/SF2-70K interaction characterized here and previously (Wu and Maniatis 1993; Kohtz et al. 1994; Jamison et al. 1995). This is also consistent with experiments of Mermoud et al. (1994), who showed that phosphatase treatment of nuclear extracts could block splicing at an early step in spliceosome assembly. Although the target proteins were not identified definitively, some (but not all) SR proteins became dephosphorylated and inhibition could be reversed by addition of a mixture of SR proteins. It is likely that RS domain phosphorylation is essential for other steps in the splicing process. For example, U1 snRNP can be dispensable for splicing in the presence of excess SR proteins (Crispino et al 1994; Tarn and Steitz 1994), indicating that SR proteins must be able to facilitate steps in splicing in addition to Ul snRNP binding to the $5^{\prime}$ splice site. This may include recruitment of the U4/U5/U6 
tri-snRNP to the spliceosome (Crispino and Sharp 1995; Tarn and Steitz 1995; Roscigno and Garcia-Blanco 1995). It will be of interest to determine the role of RS domain phosphorylation in these and other interactions requiring SR proteins.

It is also possible that unphosphorylated ASF/SF2 was inactive in our in vitro assay because it became sequestered by binding to the added random RNA. But this could also be physiologically important, for example, if nuclear speckles are storage sites for underphosphorylated SR proteins, as discussed above. It is noteworthy that the random RNA inhibits phosphorylation of ASF/ SF2 by endogenous kinases in the extract. This presumably means that when the RS domain is bound to RNA, the target serines become less accessible to the relevant kinases. This observation adds the potential for another layer of control in regulating SR protein activity. The relative local concentrations of SR proteins and RNA (as well as kinases and phosphatases) might thus determine the phosphorylation status, and hence activity, of SR proteins. In any event, our studies have shown that phosphorylation of the ASF/SF2 RS domain can have a significant effect on the protein's ability to function in splicing, likely by influencing its interactions with RNA as well as with protein.

\section{Materials and methods}

\section{Plasmid construction}

Plasmids were constructed by standard cloning procedures (Sambrook et al. 1989). Vectors encoding GST fusion proteins were constructed by inserting the coding region of ASF/SF2 or its derivatives at the carboxyl terminus of the GST coding region in pGEX-2TK (Promega). For GST-ASF, the ASF/SF2 coding sequence (BamHI-HincII fragment) from pDS-ASF (Ge et al. 1991) was ligated into BamHI and SmaI sites of pGEX-2TK. GST- $\Delta$ RS was constructed by inserting the BamHI-ApaI (bluntended) fragment of pDS-ASF into pGEX-2TK. For GST-RS, the ApaI (blunt-ended)-HincIl fragment from pDS-ASF was cloned into the SmaI site of pGEX-2TK.

\section{Expression and purification of recombinant proteins}

The GST-ASF derivatives, GST-ClkK and GST-ClkR proteins, were expressed in and purified from E. coli essentially as described (Um et al. 1995). E. coli cells (JM101; $100 \mathrm{ml}$ ) transformed with the appropriate plasmids were induced with $1 \mathrm{~mm}$ IPTG at $15^{\circ} \mathrm{C}$ overnight. Cells were harvested by centrifugation at $6000 \mathrm{rpm}$ for $10 \mathrm{~min}$ in an SS34 rotor, washed, resuspended in $4 \mathrm{ml}$ of NETN $(20 \mathrm{~mm}$ Tris at $\mathrm{pH} 8.0,100 \mathrm{~mm} \mathrm{NaCl}, 0.5 \%$ NP-40, $0.5 \mathrm{~mm}$ EDTA) and sonicated for $3 \times 1 \mathrm{~min}$. Supernatants were passed three times over $1 \mathrm{ml}$ of glutathione-agarose columns, which were washed first with $10 \mathrm{ml}$ of NETN and then with $5 \mathrm{ml}$ of NETN without NP-40. Bound proteins were eluted with $3 \mathrm{ml}$ of elution buffer $(20 \mathrm{~mm}$ glutathione, $100 \mathrm{~mm}$ Tris at $\mathrm{pH} 8.0,120 \mathrm{~mm} \mathrm{NaCl}, 0.5 \mathrm{~mm}$ EDTA $)$. Fractions $(0.5 \mathrm{ml}$ each) containing eluted proteins were pooled and dialyzed twice against buffer D (Dignam et al. 1983) for $2 \mathrm{hr}$ each at $4^{\circ} \mathrm{C}$. Histagged ASF/SF2 was expressed and purified as described /Ge et al. 1991). Protein concentrations were determined by the Bradford method.

\section{Protein phosphorylation}

GST fusion proteins (100 $\mu \mathrm{g}$ ) and His-tagged ASF/SF2 (100 $\mu \mathrm{g})$ were phosphorylated by incubation with $1 \mathrm{ml}$ of HeLa S100 extract under splicing conditions at $30^{\circ} \mathrm{C}$ for $30 \mathrm{~min} / \mathrm{Ge}$ et al. 1991). Mock phosphorylation was performed without the addition of ATP, creatine phosphate, and $\mathrm{MgCl}_{2}$. Phosphorylation of GST-ASF using GST-Clk was performed as described (Colwill et al. 1996). For each reaction, $3 \mu \mathrm{g}$ of GST-ASF was incubated with $200 \mathrm{ng}$ of GST-ClkK or GST-ClkR in phosphorylation buffer (40 mM HEPES at $\mathrm{pH} 7.5,2 \mathrm{mM} \mathrm{ATP,} 2 \mathrm{~mm} \mathrm{MgCl}_{2}$ ) at $30^{\circ} \mathrm{C}$ for $1 \mathrm{hr}$. All phosphorylated and mock-phosphorylated proteins were repurified, as described above.

\section{GST binding assays}

Purified GST, GST-ASF, GST- $\Delta$ RS and GST-RS ( $3 \mu \mathrm{g}$ each) were bound to $20 \mu \mathrm{l}$ of glutathione-agarose beads in NETN for $30 \mathrm{~min}$ at $4^{\circ} \mathrm{C}$. The beads were first washed three times with NETN-HS (NETN with high salt, $1 \mathrm{M} \mathrm{NaCl}$ ), followed by three washes with NETN $(0.1 \mathrm{M} \mathrm{NaCl})$. U1 $70 \mathrm{~K}$ was produced by in vitro translation using TNT rabbit reticulocyte lysate (Promega) and labeled with $\left.{ }^{35} \mathrm{~S}\right]$ methionine. For each binding reaction, 2 $\mu \mathrm{l}$ of $\mathrm{Ul} 70 \mathrm{~K}$ translation mixture was used, and assays were performed in $200 \mu \mathrm{l}$ of NETN at $4^{\circ} \mathrm{C}$. Beads containing bound proteins were washed three times with NETN, or as indicated. For RNase treatment, beads were incubated in $200 \mu \mathrm{l}$ of NETN with $10 \mu \mathrm{g} / \mathrm{ml}$ of RNase A at room temperature for $15 \mathrm{~min}$, and washed again three times with NETN. Proteins were eluted by boiling for $5 \mathrm{~min}$ in SDS-loading buffer. Eluted proteins were resolved on $7 \%$ SDS-PAGE. Gels were fixed and dried, and labeled proteins were visualized by fluorography. For Western blotting, proteins were separated on $10 \%$ SDS-PAGE and electroblotted on to Protran membranes (Schleicher \& Schuell). Blots were probed with mAb 104 (Roth et al. 1990) and antimouse IgM secondary antibodies. Signals were detected using the ECL reagent (Amersham). To analyze interactions with snRNPs, beads containing GST fusion proteins were incubated with $50 \mu \mathrm{l}$ of HeLa $\$ 100$ extract under splicing conditions /Ge et al. 1991). Following washing, bound fractions were deproteinized by proteinase $\mathrm{K}(50 \mu \mathrm{g} / \mathrm{ml})$ at $37^{\circ} \mathrm{C}$ for $15 \mathrm{~min}$ followed by phenol-chloroform extraction, and RNA was recovered by ethanol precipitation. RNAs were separated on $10 \%$ polyacrylamide gels containing $8 \mathrm{M}$ urea and electroblotted to Nitran membranes (Schleicher \& Schuell). Blots were hybridized with $\left[\alpha{ }^{32} \mathrm{P}\right]$ ATP-labeled antisense U1, U2, and U4 snRNA probes (Hall and Konarska 1992) in hybridization solution (6× SSPE, $10 \times$ Denhardt's, $0.1 \%$ SDS, $0.05 \%$ NP- 40 , and $50 \mu \mathrm{g} / \mathrm{ml}$ of tRNA) at $50^{\circ} \mathrm{C}$ for $20 \mathrm{hr}$, and washed three times in $6 \times \mathrm{SSPE} /$ $0.1 \%$ SDS at $50^{\circ} \mathrm{C}$. Signals were detected by autoradiography.

\section{In vitro splicing}

Tat pre-mRNA substrate was prepared essentially as described (Krainer et al. 1990a). Briefly, plasmid pSP64-tat23 (gift of A. Krainer, Cold Spring Harbor Laboratory) was linearized with $B a m H I$ and in vitro-transcribed with SP6 RNA polymerase in the presence of $\left[\alpha-{ }^{32} \mathrm{P}\right] \mathrm{GTP}$. The RNA was purified from a $6 \%$ polyacrylamide $-8 \mathrm{M}$ urea gel. In vitro splicing was performed as described (Ge and Manley 1990), except only $3 \mu$ l of nuclear extract was used. Splicing was carried out at $30^{\circ} \mathrm{C}$ for $2 \mathrm{hr}$. For ASF/SF2-supplemented reactions, $300 \mathrm{ng}$ of phosphorylated or mock phosphorylated His-ASF/SF2 was added to splicing reactions. Random RNA (76 nucleotides) was generated by in vitro transcription of a PCR-amplified sythetic template containing 20 random nucleotides downstream of $\mathrm{T} 7$ promoter /Tacke and 
Manley 1995). The indicated amounts of RNA were mixed with phosphorylated or mock-phosphorylated His-ASF/SF2 and incubated on ice for $5 \mathrm{~min}$. Reaction mixtures $(25 \mu \mathrm{l})$ were completed, and splicing was carried out at $30^{\circ} \mathrm{C}$ for $2 \mathrm{hr}$. Splicing products were deproteinized and analyzed on $6 \%$ polyacrylamide gels containing $8 \mathrm{~m}$ urea.

\section{Acknowledgments}

We thank Drs. A. Krainer, M. Konarska, K. Colwill, and T. Pawson for plasmids. We are grateful to R. Tacke, J. Prasad, and K.G.K. Murthy for advice and to members of the Manley laboratory for helpful discussions. We also thank L. Ge for excellent technical support and M. Riley for help with the manuscript. This work was supported by National Institutes of Health grant GM 48259.

The publication costs of this article were defrayed in part by payment of page charges. This article must therefore be hereby marked "advertisement" in accordance with 18 USC section 1734 solely to indicate this fact.

\section{References}

Amrein, H, M.L. Hedley, and T. Maniatis. 1994. The role of specific protein-RNA and protein-protein interactions in positive and negative control of pre-mRNA splicing by transformer 2. Cell 76: 735-746.

Cáceres, J.F. and A.R. Krainer. 1993. Functional analysis of premRNA splicing factor SF2/ASF structural domains. EMBO $J$. 12: 4715-4726.

Cardinali, B, P.T.W. Cohen, and A.I. Lamond. 1994. Protein phosphatase 1 can modulate alternative $5^{\prime}$ splice site selection in a HeLa splicing extract. FEBS Lett. 352: 276-280.

Colwill, K., T. Pawson, B. Andrews, J. Prasad, J.L. Manley, I.C. Bell, and P.I. Duncan. 1996. The Clk/Sty protein kinase phosphorylates SR splicing factors and regulates their intracellular distribution. EMBO J. 15: 265-275.

Crispino, J.D. and P.A. Sharp. 1995. A U6 snRNA: Pre-mRNA interaction can be rate-limiting for U1-independent splicing. Genes \& Dev. 9: 2314-2323.

Crispino, J.D., B.J. Blencowe, and P.A. Sharp. 1994. Complementation by SR proteins of pre-mRNA splicing reactions depleted of U1 snRNP. Science 265: 1866-1869.

Dignam, J.D., R.M. Lebovitz, and R.G. Roeder. 1983. Accurate transcription initiation by RNA polymerase II in a soluble extract from isolated nuclei. Nucleic Acids Res. 11: 1475-1489.

Eperon, I.C., D.C. Ireland, R.A. Smith, A. Mayeda, and A.R. Krainer. 1993. Pathways for selection of $5^{\prime}$ splice sites by U1 snRNPs and SF2/ASF. EMBO /. 12: 3607-3617.

$\mathrm{Fu}$, X.D. and T. Maniatis. 1992. Isolation of a complementary DNA that encodes the mammalian splicing factor SC35. Science 256: 535-538.

-1993. Specific commitment of different pre-mRNAs to splicing by single SR proteins. Nature 365: 82-85.

_ 1995 . The superfamily of arginine/serine-rich splicing factors. RNA 1: 663-680.

Ge, H. and J.L. Manley. 1990. A protein factor, ASF, controls cell-specific alternative splicing of SV40 early pre-mRNA in vitro. Cell 62: 25-34.

Ge, H, P. Zuo, and J.L. Manley. 1991. Primary structure of the human splicing factor ASF reveals similarities with Drosophila regulators. Cell 66: 373-382.

Green, M.R. 1991. Biochemical mechanisms of constitutive and regulated pre-mRNA splicing. Annu. Rev. Cell Biol. 7: 559-599.
Gui, J.F., W.S. Lane, and X.D. Fu. 1994. A serine kinase regulates intracellular localization of splicing factors in the cell cycle. Nature 369: 678-682.

Hall, K.B. and M.M. Konarska. 1992. The 5' splice site consensus RNA oligonucleotide induces assembly of U2/U4/U5/ U6 small nuclear ribonucleoprotein complexes. Proc. Natl. Acad. Sci. 89: 10969-10973.

Jamison, S.F., A. Crow, and M.A. Garcia-Blanco. 1992. The spliceosome assembly pathway in mammalian extracts. Mol. Cell. Biol. 12: 4279-4287.

Jamison, S.F., Z. Pasman, J. Wang, C. Will, R. Lührmann, J.L. Manley, and M.A. Garcia-Blanco. 1995. Ul snRNP-ASF/SF2 interaction and $5^{\prime}$ splice site recognition: Characterization of required elements. Nucleic Acids Res. 23: 3260-3267.

Kohtz, J.D., S.F. Jamison, C.L. Will, P. Zuo, R. Lührmann, M.A. Garcia-Blanco, and J.L. Manley. 1994. Protein-protein interactions and 5 '-splice site recognition in mammalian mRNA precursors. Nature 368: 119-124.

Krainer, A.R., G.C. Conway, and D. Kozak. 1990a. Purification and characterization of pre-mRNA splicing factor SF2 from HeLa cells. Genes \& Dev. 4: 1158-1171.

- $1990 \mathrm{~b}$. The essential pre-mRNA splicing factor SF2 influences $5^{\prime}$ splice site selection by activating proximal sites. Cell 62: 35-42.

Krainer, A.R., A. Mayeda, D. Kozak, and G. Binns. 1991. Functional expression of cloned human splicing factor SF2: Homology to RNA-binding proteins, U170K and Drosophila splicing regulators. Cell 66: 383-394.

Krämer, A. 1996. The structure and function of proteins involved in mammalian pre-mRNA splicing. Annu. Rev. Biochem. 65: 367-409.

Lutz, C.S., K.G.K. Murthy, N. Schek, J.B. O'Connor, J.L. Manley, and J.C. Alwine. 1996. Interaction between the U1 snRNP-A protein and the $160-\mathrm{kD}$ subunit of cleavage-polyadenylation specificity factor increases polyadenylation efficiency in vitro. Genes \& Dev. 10: 325-337.

Manley, J.L. and R. Tacke. 1996. SR proteins and splicing control. Genes \& Dev. 10: 1569-1579.

Mermoud, J.E., P. Cohen, and A.I. Lamond. 1992. Ser/Thr-specific protein phosphatases are required for both catalytic steps of pre-mRNA splicing. Nucleic Acids Res. 20: 5263-5269.

- 1994. Regulation of mammalian spliceosome assembly by a protein phosphorylation mechanism. EMBO $I$. 13: $5679-5688$.

Michaud, S. and R. Reed. 1991. An ATP-independent complex commits pre-mRNA to the mammalian spliceosome assembly pathway. Genes \& Dev. 5: 2534-2546.

Moore, M.J., C.C. Query, and P.A. Sharp. 1993. Splicing of precursors to messenger RNAs by the spliceosome. In The RNA world (ed. R.F. Gesteland and J.F. Atkins), pp. 303-358. Cold Spring Harbor Laboratory Press, Cold Spring Harbor, NY.

Mount, S.M. 1982. A catalogue of splice junction sequences. Nucleic Acid Res. 10: 459-472.

Perutz, M.F., R. Staden, L. Moens, and I. De Baere. 1993. Polar zippers. Curr. Biol. 3: 249- 253.

Roscigno, R.F. and M.A. Garcia-Blanco. 1995. SR proteins escort U4/6.U5 tri-snRNP to the spliceosome. RNA 1: 692-706.

Roth, M.B., C. Murphy, and J.G. Gall. 1990. A monoclonal antibody that recognizes a phosphorylated epitope stains lampbrush chromosome loops and small granules in the amphibian germinal vesicle. I. Cell. Biol. 111: 2217-2223.

Roth, M.B., A.M. Zahler, and J.A. Stolk. 1991. A conserved family of nuclear phosphoprotein is localized to sites of polymerase II transcription. J. Cell. Biol. 115: 587-596.

Ruby, S.W. and J. Abelson. 1988. An early hierarchic role of U1 small nuclear ribonucleoprotein in spliceosome assembly. 
Science 242: 1028-1035.

Sambrook, J., E.F. Fritsch, and T. Maniatis. 1989. Molecular cloning: A laboratory manual, 2nd edition. Cold Spring Harbor Laboratory Press, Cold Spring Harbor, NY.

Seraphin, B.L. and M. Rosbash. 1989. Identification of functional U1 snRNA-pre-mRNA complexes committed to spliceosome assembly and splicing. Cell 59: 349-358.

Seraphin, B.L., L. Kretzner, and M. Rosbash. 1988. A Ul snRNA: pre-mRNA base pairing interaction is required early in yeast spliceosome assembly but does not uniquely define the $5^{\prime}$ cleavage site. EMBO J. 7: 2533-2538.

Siliciano, P.G. and C. Guthrie. 1988. 5'-Splice site selection in yeast: Genetic alterations in base-pairing with $U 1$ reveal additional requirements. Genes \& Dev. 2: 1258-1267.

Spector, D.L. 1993. Macromolecular domains within the cell nucleus. Annu. Rev. Cell. Biol. 9: 265-315.

Staknis, D. and R. Reed. 1994. SR proteins promote the first specific recognition of pre-mRNA and are present together with the U1 small nuclear ribonucleoprotein particle in a general splicing enhancer complex. Mol. Cell. Biol. 14: $7670-7682$.

Sun, Q., A. Mayeda, R.K. Hampson, A.R. Krainer, and F.M. Rottman. 1993. General splicing factor SF2/ASF promotes alternative splicing by binding to an exonic splicing enhancer. Genes \& Dev. 7: 2598-2608.

Tacke, R. and Manley, J.L. 1995. The human splicing factors ASF/SF2 and SC35 possess different, functionally significant RNA binding specificities. EMBO T. 14: 3540-3551.

Tacke, R., Y. Chen, and J.L. Manley. 1997. Sequence-specific RNA binding by an SR protein requires RS domain phosphorylation: Creation of an SRp40-specific splicing enhancer. Proc. Natl. Acad. Sci. (in press).

Tarn, W.Y. and J.A. Steitz. 1994. SR proteins can compensate for the loss of U1 snRNP functions in vitro. Genes \& Dev. 8: 2704-2717.

- 1995. Modulation of $5^{\prime}$ splice site choice in pre-messenger RNA by two distinct steps. Proc. Natl. Acad. Sci. 92: 2504-2508.

Tazi, J., M.C. Daugeron, G. Cathala, C. Brunel, and P. Jeanteur. 1992. Adenosine phosphorothioates (ATP $\alpha$ S and ATP $\gamma$ S) differentially affect the two steps of mammalian pre-mRNA splicing. I. Biol. Chem. 267: 4322-4326.

Tazi, J., U. Kornstadt, F. Rossi, P. Jeanteur, G. Cathala, C. Brunel and R. Lührmann. 1993. Thiophosphorylation of U1$70 \mathrm{~K}$ protein inhibits pre-mRNA splicing. Nature 363: 283286.

Tian, M. and T. Maniatis. 1993. A splicing enhancer complex controls alternative splicing of doublesex pre-mRNA. Cell 74: 105-114.

Um, M., C. Li, and J.L. Manley. 1995. The transcriptional repressor Even-skipped interacts directly with the TATA binding protein. Mol. Cell. Biol. 15: 5007-5016.

Valcárcel, J. and M.R. Green. 1996. The SR protein family: Pleiotropic functions in pre-mRNA splicing. Trends Biochem. Sci. 21: 296-301.

Valcárcel, J., R.K. Gaur, R. Singh, and M.R. Green. 1996. Interaction of $U 2 \mathrm{AF}^{65} \mathrm{RS}$ region with pre-mRNA branch point and promotion of base pairing with U2 snRNA. Science 273: 1706-1709.

Wang, J., Y. Takagaki, and J.L. Manley. 1996. Targeted disruption of an essential verterate gene: ASF/SF2 is required for cell viability. Genes \& Dev. 10: 2588-2599.

$\mathrm{Wu}$, J.Y. and T. Maniatis. 1993. Specific interactions between proteins implicated in splice site selection and regulated alternative splicing. Cell 75: 1061-1070.

Zahler, A.M. and M.B. Roth. 1995. Distinct functions of SR proteins in recruitment of U1 small nuclear ribonucleoprotein to alternative $5^{\prime}$ splice sites. Proc. Natl. Acad. Sci. 92: 2642-2646.

Zahler, A.M., W.S. Lane, J.A. Stolk, and M.B. Roth. 1992. SR proteins: A conserved family of pre-mRNA splicing factors. Genes \& Dev. 6: 837-847.

Zahler, A.M., K.M. Neugenbauer, W.S. Lane, and M.B. Roth. 1993. Distinct functions of SR proteins in alternative premRNA splicing. Science 260: 219-222.

Zamore, P.D., J.G. Patton, and M.R. Green. 1992. Cloning and domain structure of the mammalian splicing factor U2AF. Nature 355: 609-614.

Zhang, W.-J. and J.Y. Wu. 1996. Functional properties of p54, a novel SR protein active in constitutive and alternative splicing. Mol. Cell. Biol. 16: 5400-5408.

Zhang, G., K.L. Taneja, R.H. Singer, and M.R. Green. 1994. Localization of pre-mRNA splicing in mammalian nuclei. $\mathrm{Na}$ ture 372: 809-812.

Zhuang, Y. and A.M. Weiner. 1986. A compensatory base change in U1 snRNA supresses a $5^{\prime}$-splice site mutation. Cell 46: 827-835.

Zuo, P. and J.L. Manley. 1993. Functional domains of the human splicing factor ASF/SF2. EMBO I. 12: 4727-4737.

. 1994. The human splicing factor ASF/SF2 can specifically recognize pre-mRNA 5' splice sites. Proc. Natl. Acad. Sci. 91: 3363-3367. 


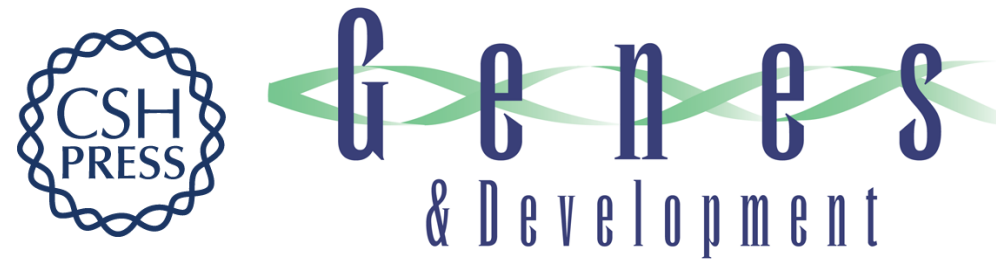

\section{Phosphorylation of the ASF/SF2 RS domain affects both protein-protein and protein-RNA interactions and is necessary for splicing.}

S H Xiao and J L Manley

Genes Dev. 1997, 11:

Access the most recent version at doi:10.1101/gad.11.3.334

References This article cites 60 articles, 27 of which can be accessed free at: http://genesdev.cshlp.org/content/11/3/334.full.html\#ref-list-1

License

Email Alerting Service

Receive free email alerts when new articles cite this article - sign up in the box at the top right corner of the article or click here.

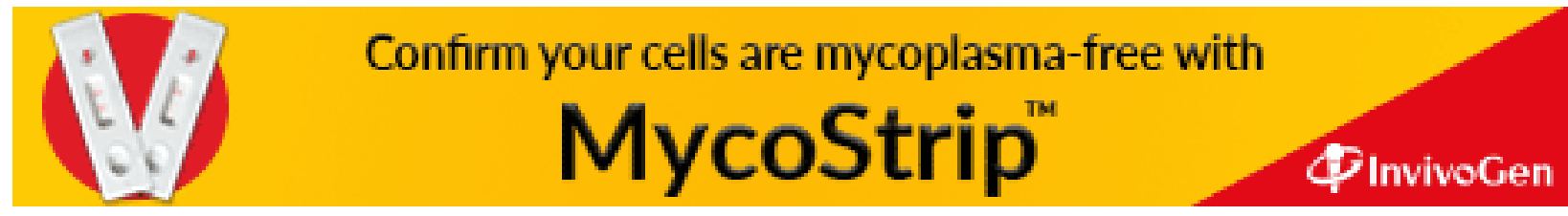

